\title{
Optimal Shift Coloring of Trees
}

\author{
Giovanni Andreatta, Luigi De Giovanni, Paolo Serafini
}

\begin{abstract}
This paper was motivated by the problem of scheduling the openings of pharmacies during week-ends and holiday periods (shifts). The problem is NP-hard in general and can be modeled as a coloring problem on a graph.In this paper we focus on the special case where the underlying graph is a tree, or, more generally, it is endowed with a tree-metric, and we provide a polynomial-time algorithm. We also provide direct optimal solutions for special trees like stars and paths.
\end{abstract}

\section{Introduction}

This paper was motivated by the problem of scheduling the openings of pharmacies during week-ends and holiday periods (see $[2,4]$ ). In practice, each pharmacy has to be assigned to one of a given number $K$ of shifts, meaning that each pharmacy will remain open 24 hours a day during one among $K$ consecutive weekends. In other words, one has to partition the set of all pharmacies into $K$ subsets, each corresponding to a particular shift. This may be viewed as a coloring problem with different colors corresponding to different shifts. In fact, it is convenient to look at the set of pharmacies as the set $V$ of vertices of an undirected graph $G(V, E)$. We also assume that the graph $G$ is connected and for every edge $\{i, j\} \in E$ a positive length $l(i, j)$ is given. Each edge $\{i, j\}$ may be thought as a direct connection (e.g. a road) between vertices (pharmacies) $i$ and $j$, and the length $l(i, j)$ is either the length of this connection or the time required to go over it. We also denote by $d_{i j}$ the shortest distance between $i$ and $j$, according to $E$ and $l$.

In the paper, we assume that the number $K$ of shifts is given and without loss of generality $2 \leq K \leq$ $|V|-1$. We let $[K]$ be the set of colours $\{1,2 \ldots, K\}$. We define $K$-Shift Coloring any coloring $\chi$ of all vertices in $V$ using all $K$ colors. Denote by $\chi(i)$ the color of the vertex $i$ and let $V_{1}, V_{2}, \ldots, V_{K}$ be the subsets of $V$ having colors $1,2, \ldots, K$.

We are interested in finding an optimal $K$-Shift Coloring $\chi$, i.e., a $K$-Shift Coloring such that the total distance

$$
D(\chi)=\sum_{i \in V} \sum_{k \in[K]} \min _{j \in V_{k}} d_{i j}
$$

is minimized.

Note that any $K$-Shift Coloring identifies a partition of $V$ into $K$ subsets.

To our knowledge, the only works devoted to this particular graph-vertex coloring problem are [2-4]. In particular, in [3] it is shown that, in general, the problem is NP-hard for $K \geq 3$ (by reduction from the domatic number problem) and polynomial for $K=2$.In this paper we consider the simplest version of such a problem where we assume that the underlying graph $G$ is a tree, or, more generally, $G$ is endowed with a tree-metric (see [5] for definition and important properties of tree-metrics). We will show that, in this case, the problem is polynomial.After introducing the notion of Perfect $K$-Shift Coloring and presenting a sufficient optimality condition that holds for arbitrary graphs (Section 2), in Section 3 we directly provide the optimal $K$-Shift Coloring of special trees like stars and paths. In Section 4, we describe the algorithm 
for finding an optimal $K$-Shift Coloring of a tree with unit distances and, in Section 5, we do the same for trees with arbitrary (positive) distances.

\section{$2 \quad$ Perfect $K$-Shift Coloring of graphs}

Given a generic graph $G=(V, E)$, a vertex $v$ and a set of $K$ vertices $Q \subseteq V$, let us denote by $D(v, Q)$ the sum of the distances from vertex $v$ to each of the $K$ vertices in $Q$. Call $K$-Core Neighborhood of $v$, and denote it by $\mathcal{C}_{v}$, any set of $K$ vertices in $G$ closest to $v$ (including vertex $v$ itself), i.e., $D\left(v, \mathcal{C}_{v}\right)=\min \{D(v, Q):|Q|=$ $K, Q \subseteq V\}$. Let us denote by $d^{*}(v)$ this minimum, i.e., $d^{*}(v)=D\left(v, \mathcal{C}_{v}\right)$. A generic $K$-Shift Coloring is full with respect to $v$ if there exists a $K$-Core Neighborhood of $v$ such that all its vertices have different colors. Let us call Full such a $K$-Core Neighborhood of $v$ and denote it by $\mathcal{F}_{v}$. Notice that, for any given $v, \mathcal{F}_{v}$ may or may not exist, and may or may not be unique. If $\mathcal{F}_{v}$ exists, then $D\left(v, \mathcal{F}_{v}\right)=d^{*}(v)$.

We remark that the notion of $K$-Core Neighborhood is independent from any coloring, while that of Full $K$-Core Neighborhood depends on the selected coloring $\chi$.

Given a graph $G$, let us call Perfect $K$-Shift Coloring of $G$ a coloring $\chi^{\sharp}$ such that $\chi^{\sharp}$ is full with respect to every vertex $v$, i.e., there exists a Full $K$-Core Neighborhood of $v$, for any vertex $v$.

Notice that a Perfect $K$-Shift Coloring of $G$ may not exist. The circuit with 4 vertices and unit distances is a simple example of a graph for which there is no Perfect 3-Shift Coloring. We will show in Sections 4 and 5 that every tree possesses a Perfect $K$-Shift Coloring.

The following theorem provides an optimality sufficient condition, holding for any graph $G$ (and not just for trees) and for any type of distances.

Theorem 1. Any Perfect $K$-Shift Coloring $\chi^{*}$ of a graph $G$ is also an optimal K-Shift Coloring of $G$.

Proof: Given any $K$-Shift Coloring $\chi$ of $G$, let $V_{1}, V_{2}, \ldots, V_{K}$ and $D(\chi)$ be defined as in Section 1 . We have: $D(\chi)=\sum_{v \in V} \sum_{k \in[K]} \min _{j \in V_{k}} d_{v j} \geq \sum_{v \in V} D\left(v, \mathcal{C}_{v}\right)=\sum_{v \in V} d^{*}(v)$ and $D\left(\chi^{*}\right)=\sum_{v \in V} D\left(v, \mathcal{F}_{v}\right)=$ $\sum_{v \in V} D\left(v, \mathcal{C}_{v}\right)=\sum_{v \in V} d^{*}(v)$. This proves that $D(\chi) \geq D\left(\chi^{*}\right)$.

Furthermore, the proof of Theorem 1 also shows the following result:

Theorem 2. If a graph $G$ possesses a Perfect $K$-Shift Coloring, then all optimal $K$-Shift Coloring of $G$ are perfect.

In the following sections we will show that every tree possesses a Perfect $K$-Shift Coloring, and how to build one.

\section{$3 \quad K$-Shift Coloring of special trees}

Let us consider the case where the graph is a tree and, as a special case, assume that $T$ is a star with unit distances. We have the following 
Proposition 1 Let $T$ be a star with unit distances and vertices numbered $1,2, \ldots, n$, where vertex 1 is the center. Any K-Shift Coloring such that vertex 1 gets color 1 and every pendant vertex gets a color between 2 and $K$, with at least one vertex per color, is a Perfect $K$-Shift Coloring of $T$ and thus it is optimal and, vice-versa, every optimal $K$-Shift Coloring of $T$ is of this form (up to a permutation of colors).

Proof: Although the proposition is a simple corollary of Theorems 1 and 2, we provide here an alternative direct proof. Let $\chi$ be any $K$-Shift Coloring of $T$. We may assume, without loss of generality, that $\chi(1)=1$. Let $m_{1}, m_{2}, \ldots, m_{K}$ be the number of pendant vertices of $T$ that are given colors $1,2, \ldots, K$ respectively. Notice that $m_{1}+m_{2}+\ldots+m_{K}=n-1$. Of course $m_{j} \geq 1$. The total distance that has to be traveled during shift 1 is

$$
D_{1}=0 \cdot\left(1+m_{1}\right)+1 \cdot \sum_{k>1} m_{k}=n-1-m_{1}
$$

and during shift $h(2 \leq h \leq K)$

$$
D_{h}=1+2 \cdot \sum_{k>1, k \neq h} m_{k}=1+2\left(n-1-m_{h}\right)
$$

so that the total distance is

$$
\begin{gathered}
n-1-m_{1}+\sum_{h>1}\left(1+2\left(n-1-m_{h}\right)\right)= \\
=n-1-m_{1}+K-1+2(n-1)(K-1)-2\left(n-1-m_{1}\right)= \\
=m_{1}-3 n+(2 n-1) K+2
\end{gathered}
$$

Evidently the total distance is minimized if and only if $m_{1}=0$, and its optimal value is equal to

$$
(2 n-1)(K-1)-(n-1)
$$

no matter what the values $m_{2}, \ldots m_{K}$ are, provided that they are all at least 1 . This proves that any optimal $K$-Shift Coloring has the form described in the statement of the theorem. It is also evident that such a $K$-Shift Coloring is perfect.

The hypothesis of unit distances can actually be relaxed, as stated in the following

Proposition 2 If $T$ is a star with arbitrary distances, and the vertices are numbered from 1 to $n$ according to their increasing distance from the center (vertex 1), then the K-Shift Coloring $\chi^{*}$ defined as

$$
\chi^{*}(v)=\min (v, K) \quad v=1, \ldots, n,
$$

is perfect and thus optimal. Furthermore, every optimal K-Shift Coloring is of this form (up to a permutation of colors).

Proof: If $v \leq K$ then the subset of vertices $\{1,2, \ldots, K\}$ is a $\mathcal{C}_{v}$, that is also a $\mathcal{F}_{v}$. If $v>K$ then the subset of vertices $\{1,2, \ldots, K-1, v\}$ is a $\mathcal{C}_{v}$, that, again, is also a $\mathcal{F}_{v}$. Hence, the given $K$-Shift Coloring is perfect and, by Theorem 1, optimal. It is also evident that any Perfect $K$-Shift Coloring must have this form (up to a permutation of colors). By Theorem 2 it follows that all optimal $K$-Shift Coloring must have this form (up to a permutation of colors). 
As a corollary of Theorem 1, we also have the following

Proposition 3 If $T$ is a path with arbitrary distances and its vertices are numbered $1,2, \ldots, n$ in a canonical way (i.e., vertex 1 is a pendant vertex and the number of edges in the path from vertex 1 to vertex $j$ is $j-1$, for any $j=2, \ldots, n$ ), then a Perfect (and optimal) $K$-Shift Coloring of $T$ is $\chi^{*}(j)=j \bmod K$.

One may wonder if every Perfect $K$-Shift Coloring of a graph has the form described in Proposition 3 . The answer is no, as the following example shows. Let $K=2$ and let $P$ be the path with 4 vertices and 3 edges where $l(1,2)=l(3,4)=1$ and $l(2,3)=2$. For this path, the following $K$-Shift Coloring: $\chi^{\sharp}(1)=\chi^{\sharp}(4)=1$ and $\chi^{\sharp}(2)=\chi^{\sharp}(3)=2$ is also perfect (and thus optimal), but it is not of the form described in Proposition 3.

\section{$4 \quad$ K-Shift Coloring of trees with unit distances}

The previous Propositions 2 and 3 seem to indicate that the order in which vertices are colored is important. This is confirmed in the general case by the following algorithm. Given a tree $T$ with unit distances, an optimal $K$-Shift Coloring of $T$ may be found in polynomial time by the following

\section{Algorithm 1}

Given a tree $T$ and a number of colors $K$, do:

1. Ordering of vertices and initial colored subtree. Choose any vertex of $T$ as root (denote it by $r$ ) and order the vertices from 1 to $n$ according to a breadth first exploration. Color the first $K$ vertices with the $K$ different colors, obtaining a colored subtree $T_{K}$. Set $m=K$.

2. Inductive construction of colored subtrees. Consider vertex $w:=m+1$ and let $T_{m+1}=T_{m} \cup\{w\}$ be the subtree containing the vertices from 1 to $m+1$. For each $k \in\{1, \ldots, K\}$, let $v_{k}$ be a vertex of $T_{m}$ with color $k$ having minimum distance from $w$. Let $z$ be a vertex with maximum distance from $w$, among $v_{1}, v_{2}, \ldots, v_{K}$. Give $w$ the same color of $z$, i.e., $\chi(m+1)=\chi(z)$.

3. Stopping criterion. Set $m=m+1$. If $m=n+1$ stop, otherwise go to Step 2 .

The algorithm produces a $K$-Shift Coloring $\chi$ and a sequence of subtrees $T_{K} \subset T_{K+1} \subset \ldots \subset T_{n}=T$. In the following - see Proof of correctness of Algorithm 1 - we are going to show that, for each $m \in$ $\{K, K+1, \ldots, n\}$, the coloring $\chi$, restricted to $T_{m}$, is optimal for $T_{m}$.

See in Figure 1 a tree colored with 4 colors according to the algorithm (each layer at the same distance from the root is colored from left to right).

Before proving the correctness of the algorithm, let us introduce the following notations, definitions and lemmas.

Notations:

- Let $P(u, v)$ denote the (unique) simple path connecting vertices $u$ and $v$. 


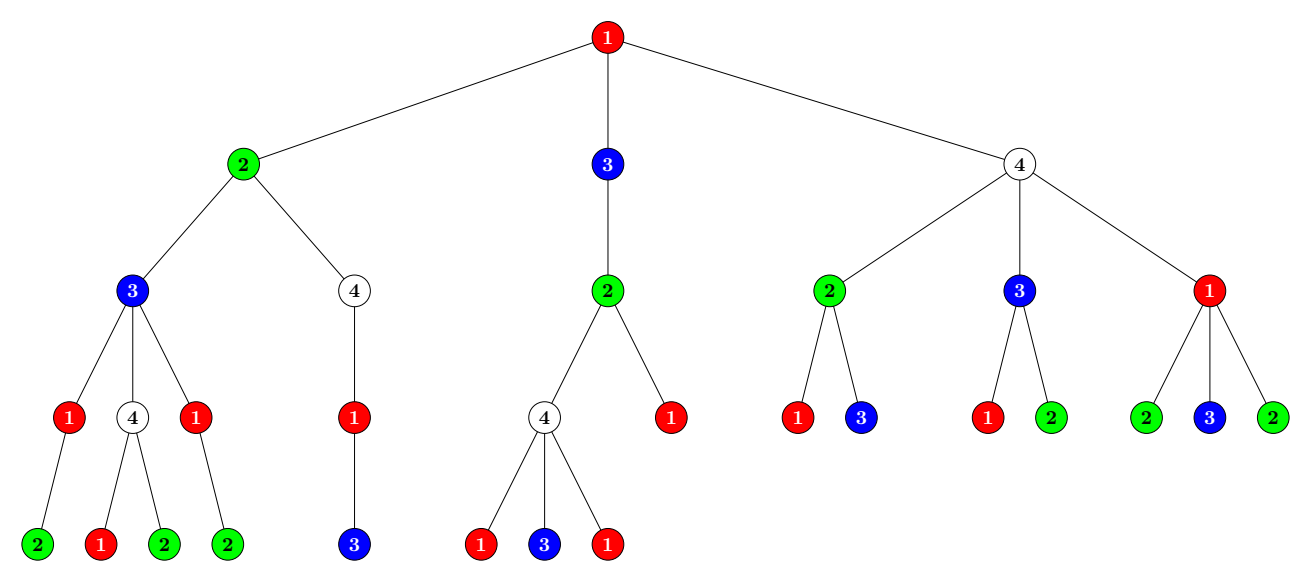

Fig. 1. An optimal 4-Shift Coloring on a sample tree

- For each $m \in\{K, K+1, \ldots, n\}$ and $v \in T_{m}$, let $\mathcal{C}_{v}^{m}$ denote any set of $K$ vertices in $T_{m}$ closest to vertex $v$, i.e., a $K$-Core Neighborhood of $v$ in $T_{m}$.

- For each $m \in\{K, K+1, \ldots, n\}$ and $v \in T_{m}$, let $\mathcal{F}_{v}^{m}$ denote any $\mathcal{C}_{v}^{m}$ such that all $K$ vertices of $\mathcal{C}_{v}^{m}$ have different colors, i.e., a Full $K$-Core Neighborhood of $v$ in $T_{m}$.

Definition 1 Given vertices $u, v$ and $w$, let us call projection of $w$ onto $P(u, v)$ the unique vertex belonging to all three simple paths $P(u, v), P(u, w)$ and $P(v, w)$.

Lemma 1 For any set of four vertices $u, v, c, g$ with $c \in P(u, v)$, either

(i) $-c$ belongs to $P(g, u)$ or

(ii) $-c$ belongs to $P(g, v)$ but not to $P(g, u)$.

Proof: Let $g^{\prime}$ be the projection of $g$ onto $P(u, v)$. If the distance from $g^{\prime}$ to $v$ is less than or equal to the distance from $c$ to $v$, then case (i) follows; otherwise, $g^{\prime}$ is closer to $u$ than $c$ and case (ii) follows.

Lemma 2 At any step of the algorithm, if $v$ and $w$ are vertices descendant of a same vertex $q$, with $v$ already colored and $w$ not yet colored, then necessarily $d_{q v} \leq d_{q w}$.

Proof: This is due to the breadth first exploration rule adopted by the algorithm for coloring the vertices of $T$.

Proof of correctness of Algorithm 1: The proof proceeds by induction on $m$, the cardinality of the subtree $T_{m}$. The coloring of $T_{K}$ is optimal because of Theorem 1. Suppose that all the subtrees created by the algorithm, up to a given $m(m \geq K)$, satisfy the hypothesis of Theorem 1 . We want to show that also $T_{m+1}$, colored according to the algorithm, satisfies the property that, for each vertex $v \in T_{m+1}$ there exists a 


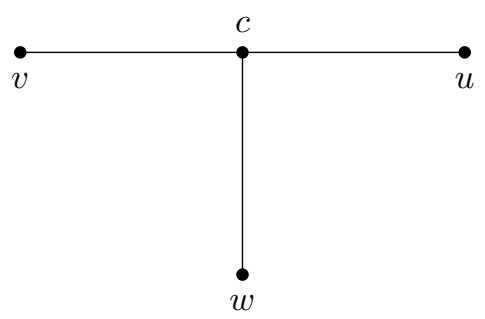

Fig. 2. Relevant vertices in the tree: $c$ is the projection of $w$ onto $P(u, v)$.

Full $K$-Core Neighborhood of $v, \mathcal{F}_{v}^{m+1} \subset T_{m+1}$, so that $T_{m+1}$ has a Perfect $K$-Shift Coloring and therefore is optimal by Theorem 1 .

Let $v$ be a generic vertex in $T_{m+1}$ and let $w$ be the vertex that belongs to $T_{m+1}$ but not to $T_{m}$. Let us distinguish between two cases:

- case 1: $v \in T_{m}$

- case 2: $v=w$.

In case 1 , we further distinguish two subcases: either (case 1.1) there exists a $\mathcal{F}_{v}^{m}$ in $T_{m}$ that is also a $\mathcal{F}_{v}^{m+1}$ in $T_{m+1}$, or else (case 1.2) no $\mathcal{F}_{v}^{m}$ in $T_{m}$ is a $\mathcal{F}_{v}^{m+1}$ in $T_{m+1}$.

In case 1.1, the induction step is obviously true for that particular $v \in T_{m}$.

In case 1.2 , choose any specific $\mathcal{F}_{v}^{m}$ and let:

$u$ be the farthest vertex from $v$ in $\mathcal{F}_{v}^{m}$,

$y$ be the vertex in $\mathcal{F}_{v}^{m}$ having color $\chi(y)=\chi(w)$, and

$z$ be defined as in Step 2, i.e., the farthest vertex from $w$ among $v_{1}, v_{2}, \ldots, v_{K}$ with $v_{i}$ being the closest vertex to $w$ among those having color $i(i=1,2, \ldots, K)$.

Since $w \in T_{m+1} \backslash T_{m}$ and $u, y$ and $z \in T_{m}$, we must have $w \neq u, y, z$ and, by definition of $u, v \neq u$. Also, by construction, $\chi(w)=\chi(y)=\chi(z)$. If $d_{v w} \geq d_{v u}$, then $\mathcal{F}_{v}^{m}$ is also a $\mathcal{F}_{v}^{m+1}$, which is not possible in case 1.2. If $\chi(u)=\chi(w)$, then $\mathcal{F}_{v}^{m} \cup\{w\} \backslash\{u\}$ is a $\mathcal{F}_{v}^{m+1}$ as required. So, in the following, assume that

$$
d_{v w}<d_{v u}
$$

and that $\chi(u) \neq \chi(w)$ which, in turn, implies that $u \neq z, y$. As for $v, y$ and $z$, it is possible that they are not all distinct. We will show (Claim 10) that starting from $\mathcal{F}_{v}^{m}$ and considering $F^{*}=\left(\mathcal{F}_{v}^{m} \cup\{w\}\right) \backslash\{y\}$ we have that $F^{*}$ is a Full $K$-Core Neighborhood of $v$ in $T_{m+1}$. The rest of the proof for case 1.2 is rather lengthy so we break it into several claims.

Let $c$ be the projection of $w$ onto $P(v, u)$ (see Figure 2). Notice that $c \neq u$, because otherwise $d_{v u} \leq d_{v w}$ contradicting (1), and that $c \neq w$ because $w$ is a leaf of $T_{m+1}$ and therefore cannot be an internal vertex of $P(u, v)$. It is possible however that $c=v$. Finally let $c^{\prime}$ be the projection of the root $r$ on the path $P(u, v)$.

Claim 1 The distance from $c$ to $u$ is strictly greater than the distance from $c$ to $w$. 


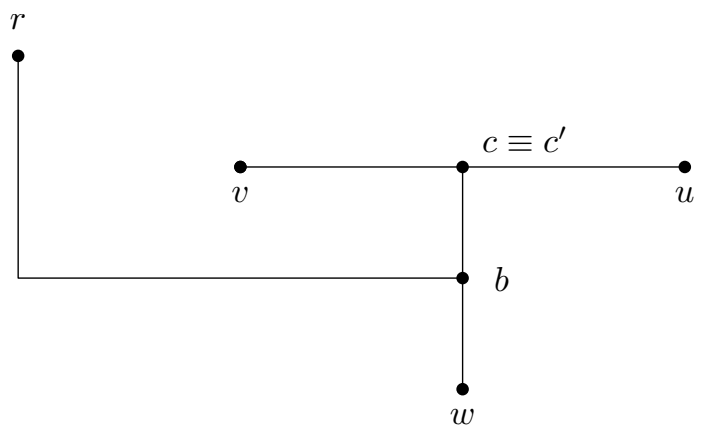

Fig. 3. Relevant vertices in the tree: $b$ is the projection of $c$ onto $P(r, w)$.

Proof of Claim 1: Since paths are on a tree we have $d_{v u}=d_{c v}+d_{c u}$ and $d_{v w}=d_{c v}+d_{c w}$. By subtracting one equality from the other and from (1) we have

$$
d_{c u}>d_{c w}
$$

Claim 2 The vertex closest to $c$ among those in $T_{m}$ having color $\chi(u)$ must be $u$ itself, or, if such a vertex is not unique, $u$ must be one of these vertices.

Proof of Claim 2: Suppose that a vertex $t$ exists having color $\chi(u)$ and having distance $d_{c t}<d_{c u}$. Then, exploiting the triangular inequality, $d_{v t} \leq d_{v c}+d_{c t}<d_{v c}+d_{c u}=d_{v u}$ meaning that $t$ is closer to $v$ than $u$, against the fact that $u \in \mathcal{F}_{v}^{m}$ and $t \notin \mathcal{F}_{v}^{m}$, since, by the inductive hypothesis, only one vertex of color $\chi(u)$ belongs to $\mathcal{F}_{v}^{m}$.

Claim 3 Vertex $c$ belongs to the path $P(r, w)$, that is, $w$ is a descendant of $c$.

Proof of Claim 3: Recall $c^{\prime}$ is the projection of the root $r$ on the path $P(u, v)$. Either $c^{\prime}=c$ or $c^{\prime} \neq c$. If $c^{\prime}=c$, let $b$ be the projection of $c$ onto $P(r, w)$ (see Figure 3). By Lemma 2, we have $d_{b u} \leq d_{b w}$. This implies $d_{c u} \leq d_{b u} \leq d_{b w} \leq d_{c w}$, contradicting inequality (2). Therefore $c^{\prime} \neq c$ and the path $P(w, c) \cup P\left(c, c^{\prime}\right) \cup P\left(c^{\prime}, r\right)$ is the (unique) path connecting $w$ with $r$. This proves that $c \in P(r, w)$.

Notice also that $c^{\prime} \in P(c, u)$ (see Figure 4), as otherwise $c^{\prime} \in P(c, v)$. In the latter case, $c \in P(r, u)$ and both $u$ and $w$ will be descendant of the same vertex $c$. By Lemma 2 , we have $d_{c u} \leq d_{c w}$, contradicting inequality (2).

Claim 4 Vertex $u$ has shortest distance from $w$ among all vertices in $T_{m}$ having color $\chi(u)$. 


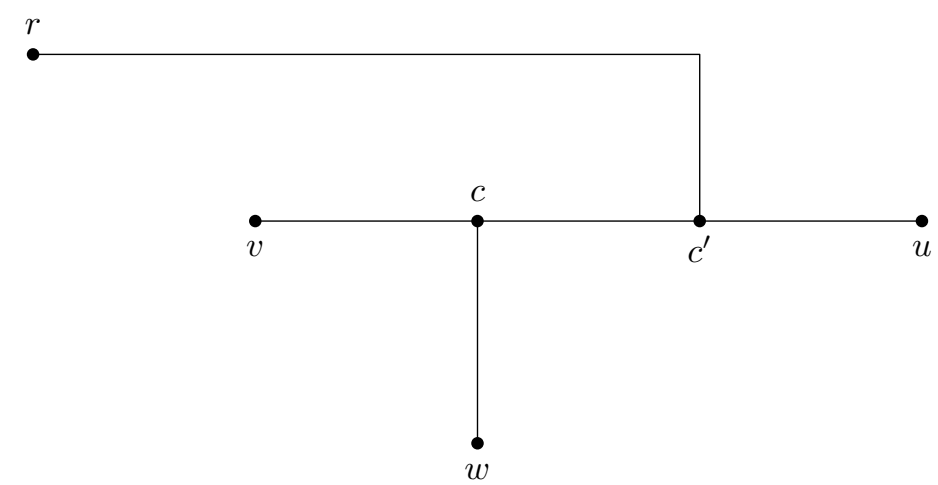

Fig. 4. Relevant vertices in the tree: $c^{\prime}$ is the projection of $r$ onto $P(u, v)$.

Proof of Claim 4: Let $x$ be a vertex in $T_{m}$ having the shortest distance from $w$ among all vertices in $T_{m}$ having color $\chi(u)$. We will show that $d_{w x}=d_{w u}$.

By Lemma 1, applied to the four vertices $r, w, c, x$, either $c$ belongs to the path connecting $x$ with $r$ or else $c$ belongs to the path connecting $w$ with $x$.

However, $c$ cannot belong to the path connecting $x$ with $r$, because otherwise both $w$ and $x$ would be descendant of $c$ and $d_{c x} \leq d_{c w}$, by Lemma 2. Then, it follows from Claim 1 that $d_{c x} \leq d_{c w}<d_{c u}$, which contradicts Claim 2, i.e., the fact that $u$ is the closest vertex to $c$ among those having color $\chi(u)$.

Therefore $c$ must be in the path connecting $w$ with $x$ and recalling that $u$ is the vertex closest to $c$ among those having color $\chi(u)$, we have: $d_{w x}=d_{w c}+d_{c x} \geq d_{w c}+d_{c u}=d_{w u}$. Since $d_{w x} \leq d_{w u}$, by definition of $x$, this proves that $d_{w u}=d_{w x}$, i.e., $u$ is the vertex (or one of the vertices) in $T_{m}$ having the shortest distance from $w$, among all vertices in $T_{m}$ having color $\chi(u)$.

From Claim 4, it follows that

$$
d_{w u} \leq d_{w z} .
$$

Claim 5 Vertex $c$ belongs to $P(w, z)$.

Proof of Claim 5: Let us first prove that $c \notin P(z, r)$. In fact, if $c \in P(z, r)$, we would have $d_{c z} \leq d_{c w}$, due to Lemma 2, and this in turn will imply by Claim 1 that $d_{c z} \leq d_{c w}<d_{c u}$ and therefore $d_{w z} \leq d_{w c}+d_{c z}<$ $d_{w c}+d_{c u}=d_{w u}$ contradicting (3). Recall that, by Claim 3, $c \in P(r, w)$. Since $c \notin P(z, r)$, then, by virtue of Lemma 1 applied to the four vertices $r, w, c$, $z$, we have $c \in P(w, z)$.

Claim 6 The vertex closest to $c$ among those in $T_{m}$ having color $\chi(w)=\chi(z)$ is $z$ itself, or, if such a vertex is not unique, $z$ is one of these vertices.

Proof of Claim 6: Suppose that a vertex $s$ exists having color $\chi(s)=\chi(z)$ and having distance $d_{c s}<d_{c z}$, and let $s^{\prime}$ be the projection of $s$ onto $P(w, z)$. Since we have just proved that $c$ belongs to $P(w, z)$, either, 


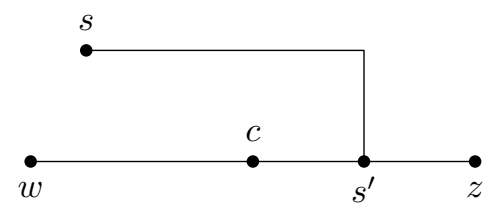

(i)

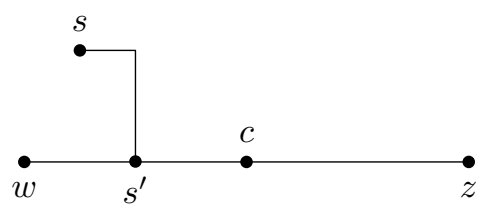

(ii)

Fig. 5. Relevant vertices in the tree: $s^{\prime}$ is the projection of $s$ onto $P(w, z)$.

(i) $s^{\prime}$ belongs to $P(c, z)$ (Figure $5 i$ ), or, (ii) $s^{\prime}$ does not belong to $P(c, z)$ (Figure 5ii). In case (i), $d_{w s}=$ $d_{w c}+d_{c s}<d_{w c}+d_{c z}=d_{w z}$, contradicting the fact that $z$ is the closest vertex to $w$ among those having color $\chi(w)$. In case (ii), $d_{w s}=d_{w s^{\prime}}+d_{s^{\prime} s} \leq d_{w c}+d_{c s}<d_{w c}+d_{c z}=d_{w z}$, again contradicting the fact that $z$ is the closest vertex to $w$ among those having color $\chi(w)$.

Claim 7 Vertices $z$ and $y$ have the same distance from $v$, and $c \in P(v, z)$.

Proof of Claim 7: Recall that $y$ is the vertex in $\mathcal{F}_{v}^{m}$ having color $\chi(y)=\chi(w)=\chi(z)$. Let us first prove that $y \neq v$. Recall that $d_{w v}<d_{w u}$, by inequality (1); $d_{w u} \leq d_{w z}$, by (3); $d_{w z} \leq d_{w y}$, by definition of $z$. Thus we have $d_{w v}<d_{w y}$. This proves that $y \neq v$. We want to prove that $d_{v y}=d_{v z}$. Let $y^{\prime}$ be the projection of $y$ onto $P(w, r)$. Since, by Claim 3, also $c \in P(w, r)$, then either (case 1.2.1) $y^{\prime}$ belongs to $P(c, w)$ (Figure 6a), else (case 1.2.2) $y^{\prime}$ belongs to $P(c, r)$ but not to $P(c, w)$ (Figures 6b or 6c). If $y^{\prime}$ belongs to $P(c, w)$, we have $d_{c y} \leq d_{c w}$, by Lemma 2 , and since $d_{c w}<d_{c u}$ by (2), we obtain $d_{c y}<d_{c u}$ which in turn implies that $d_{w y} \leq d_{w c}+d_{c y}<d_{w c}+d_{c u}=d_{w u} \leq d_{w z}$ (by (3)), contradicting the hypothesis that $z$ is the closest vertex to $w$ among those having color $\chi(w)$. Thus case (1.2.2) holds, that is, $y^{\prime} \in P(c, r)$. Now, let us consider again $c^{\prime}$, the projection of $r$ onto $P(u, v)$ : as we have seen (refer again to Figures $6 \mathrm{~b}$ and $6 \mathrm{c}$ ), $c^{\prime} \in P(c, u)$, which implies that $c \in P(v, r)$ and, since $y^{\prime} \in P(c, r)$, we have $c \in P(v, y)$ and, hence, $d_{v y}=d_{v c}+d_{c y}$. Since $d_{v z} \leq d_{v c}+d_{c z} \leq d_{v c}+d_{c y}$, thanks to Claim 6 , and $d_{v c}+d_{c y}=d_{v y} \leq d_{v z}$, we conclude that $d_{v y}=d_{v z}$ and, from $d_{v y}=d_{v c}+d_{c y}$, it follows that $c \in P(v, z)$.

Claim 8 The set $F^{\prime}=\left(\mathcal{F}_{v}^{m} \cup\{z\}\right) \backslash\{y\}$ is also a Full $K$-Core Neighborhood of $v$ in $T_{m}$.

Proof of Claim 8: This is obviously true since $\chi(y)=\chi(z)$ and $d_{v y}=d_{v z}$ as proved in Claim 7. Notice that, as shown in the proof of Claim $7, y \neq v$.

Claim 9 Vertex $z$ is one of the farthest vertices from $v$ in $F^{\prime}$.

Proof of Claim 9: Since $d_{v z}=d_{v y}$ by Claim 7 and $d_{v y} \leq d_{v u}$, by definition of $u$, we have

$$
d_{v z} \leq d_{v u}
$$


Recalling the definition of $z$ and Claim 4, we have: $d_{w z} \geq d_{w u}$. Notice that $c \in P(v, u)$ and $c \in P(w, u)$ by definition of $c ; c \in P(w, z)$ by Claim 5 , and $c \in P(v, z)$ by Claim 7. Therefore, from $d_{w z} \geq d_{w u}$, by adding $d_{v c}-d_{w c}$ to both members we obtain: $d_{v z} \geq d_{v u}$ that, together with inequality (4) implies: $d_{v z}=d_{v u}$.

Recalling that $u$ is the farthest vertex from $v$ in $\mathcal{F}_{v}^{m}$ and the definition of $F^{\prime}$, we have Claim 9.

Claim 10 The set $F^{*}=\left(\mathcal{F}_{v}^{m} \cup\{w\}\right) \backslash\{y\}$ is a Full $K$-Core Neighborhood of $v$ in $T_{m+1}$.

Proof of Claim 10: Notice that $F^{*}=\left(\mathcal{F}_{v}^{m} \cup\{w\}\right) \backslash\{y\}=\left(F^{\prime} \cup\{w\}\right) \backslash\{z\}$. The set $F^{*}$ is a $K$-Core Neighborhood of $v$ in $T_{m+1}$, because $d_{v w}<d_{v u}$, by (1), and $d_{v u}=d_{v z}$, by Claim 9 . Since all $K$ vertices in $F^{*}$ have now different colors, we have that $F^{*}$ is a Full $K$-Core Neighborhood of $v$ in $T_{m+1}$.

This concludes the proof of correctness of the Algorithm in Case 1.2.

To complete the proof we have to consider Case 2, i.e., we have to show that there exists in $T_{m+1}$ a Full $K$-Core Neighborhood of $w$, the only vertex belonging to $T_{m+1}$, but not to $T_{m}$. Let $f$ be the father of $w$ in $T_{m+1}$. Since $f \in T_{m}$, then by the previous proof of Case 1 , there exists a Full $K$-Core Neighborhood $\mathcal{F}_{f}^{m+1}$ in $T_{m+1}$. Since $d(f, w)=1$ it follows that $\mathcal{F}_{f}^{m+1}$ contains $w$, or at least there exists one $\mathcal{F}_{f}^{m+1}$ that contains $w$. If $v_{k}$ is the vertex of color $k(k \neq \chi(w))$ belonging to $\mathcal{F}_{f}^{m+1}$, it means that $v_{k}$ is the closest vertex to $f$ among those having color $k$. Noticing that $d(w, v)=d(f, v)+1$, for any $v$ in $T_{m}$, it follows that $v_{k}$ is also the closest vertex to $w$ among those having color $k$. Therefore $\mathcal{F}_{f}^{m+1}$ is also a Full $K$-Core Neighborhood of $w$.

\section{$5 \quad K$-Shift Coloring of trees with arbitrary distances and graphs endowed with a tree metric}

Given a tree $T$ with arbitrary (positive) distances, an optimal $K$-Shift Coloring of $T$ can be found by the following

\section{Algorithm 2}

Given a weighted tree $T$ and a number of colors $K$, do:

\section{Step 1:}

1. Ordering of vertices and initial colored subtree. Choose any vertex of $T$ as root (denote it by $r$ ) and order the vertices from 1 to $n$ according to their increasing distance from the root $r$; break ties arbitrarily. Color the first $K$ vertices with the $K$ different colors, obtaining a colored subtree $T_{K}$. Set $m=K$.

2. Inductive construction of colored subtrees. Consider vertex $w:=m+1$ and let $T_{m+1}=T_{m} \cup\{w\}$ be the subtree containing the vertices from 1 to $m+1$. For each $k \in\{1, \ldots, K\}$, let $v_{k}$ be a vertex of $T_{m}$ with color $k$ having minimum distance from $w$. Let $z$ be a vertex with maximum distance from $w$, among $v_{1}, v_{2}, \ldots, v_{K}$. Give $w$ the same color of $z$, i.e., $\chi(m+1)=\chi(z)$. 
3. Stopping criterion. Set $m=m+1$. If $m=n+1$ stop, otherwise go to Step 2 .

The proof of correctness is analogous to the one of the previous algorithm and thus omitted. The main difference is the reason why, given a vertex $q$ in the path between $w$ and $r$, any vertex $a$ in $T_{m}$ that is a descendant of vertex $q$ has distance from $q$ that satisfies the inequality $d_{q a} \leq d_{q w}$ : this is guaranteed by the search criterion adopted by the algorithm. This property is the analogous of Lemma 2 in Section 4 .

A second important difference in each iteration concerns the vertex $w$ that belongs to $T_{m+1}$ but not to $T_{m}$. In the case of arbitrary positive distances it is no longer necessarily true that $\mathcal{F}_{w}^{m+1}$ coincides with

one $\mathcal{F}_{f}^{m+1}$, where $f$ is the father of vertex $w$. This happens when $f$ and $w$ are very far apart. In this case, consider a Full $K$-Core Neighborhood of $f: \mathcal{F}_{f}^{m}=v_{1}, v_{2}, \ldots, v_{K}$. This means that $v_{1}, v_{2}, \ldots, v_{K}$ have all different colors. Since $d\left(w, v_{k}\right)=d(w, f)+d\left(f, v_{k}\right)$ for all $k=1,2, \ldots, K$, it follows that $v_{1}, v_{2}, \ldots, v_{K}$ are the closest vertices to $w$, besides $w$ itself. Defining $z$ as the farthest vertex from $w$ among $v_{1}, v_{2}, \ldots, v_{K}$ it follows that $\left(\left\{v_{1}, v_{2}, \ldots, v_{K}\right\} \cup\{w\}\right) \backslash\{z\}$ is a $K$-Core Neighborhood of $w$ that is also a Full $K$-Core Neighborhood of $w$, since all vertices $v_{1}, v_{2}, \ldots, v_{K}$ have different colors and the color of $w$ is the same as that of $z$.

Proposition 4 For any tree $T$ with positive distances, there is an optimal $K$-Shift Coloring where $\chi(i) \neq$ $\chi(j)$ for all edges $\{i, j\} \in E$.

Proof: The optimal coloring generated by the algorithm does satisfy the stated property.

Notice that this property, valid for trees, is not true for a general graph as shown by the following simple counter-example. Given any 2-Shift Coloring of a cycle of length 3, there is an edge with its extremes having the same color. The following corollary, that could also be derived from Algorithm 2, is an immediate consequence of Proposition 4.

Corollary 1 Given an arbitrary tree $T$, the classic 2-Coloring of $T$ also provides an optimal 2-Shift Coloring of $T$.

Consider now a graph $G=\left(V^{G}, E^{G}\right)$ endowed with a tree metric $d^{G}$. This means that there exists a tree $T=\left(V^{T}, E^{T}\right)$ with $V^{T}=V^{G}=V$ having a metric $d^{T}$ such that $d_{i j}^{G}=d_{i j}^{T}$ for any couple of vertices $i, j \in V$.

Given an arbitrary graph $G$ endowed with a tree metric, an optimal $K$-Shift Coloring of $G$ may be obtained by coloring its vertices according to an optimal $K$-Shift Coloring of the associated tree $T$. The proof is trivial and thus omitted.

\section{Acknowledgment}

The authors want to thank an anonymous referee for the remarks that greatly improved the quality of the paper, and, in particular, for pointing out the result stated in Theorem 2. 


\section{References}

1. R. K. Ahuja, T. L. Magnanti and J. B. Orlin, Network flows: Theory, algorithms, and applications, Prentice Hall, Englewood Cliffs, NJ (1993).

2. G. Andreatta, L. De Giovanni and P. Serafini, "Scheduling Pharmacy Openings", in: Proceedings of ECCO 2012 - 25th Conference of European Chapter on Combinatorial Optimization, April 26-28, 2012, Antalya, Turkey (2012).

3. G. Andreatta, L. De Giovanni and P. Serafini, "Optimal Shift Partitioning of Pharmacies", Working Paper (2013).

4. Ö. Özpeynirci and E. Ağlamaz, "Mathematical Models for Pharmacy Duty Scheduling", in: Proceedings of ECCO 2012 - 25th Conference of European Chapter on Combinatorial Optimization, April 26-28, 2012, Antalya, Turkey (2012).

5. A. N. Patrinos and S. L. Hakimi, "The distance matrix of a graph and its tree realization", Quarterly of Applied Mathematics, 30, 255-269 (1972). 


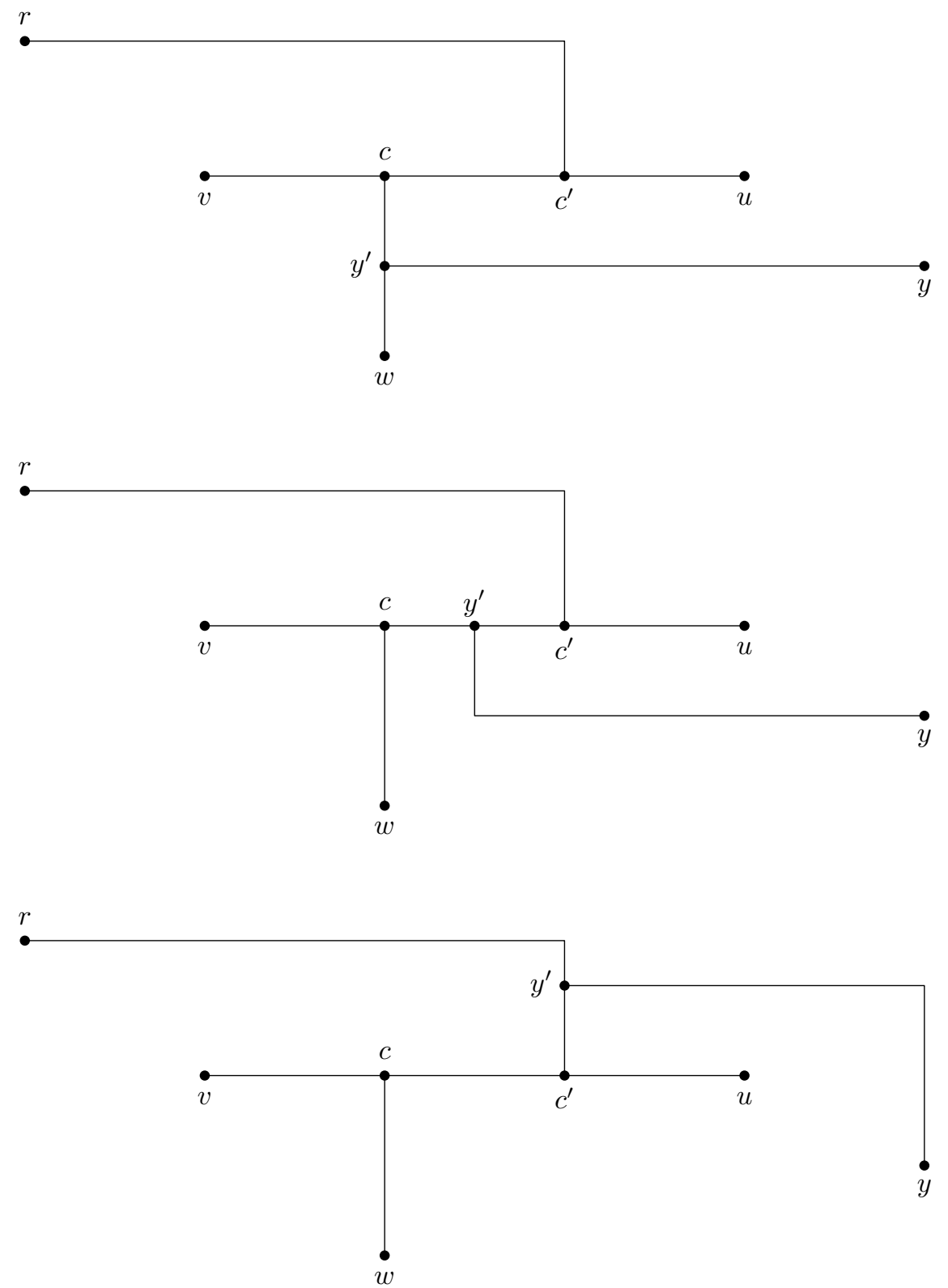

(a)

(b)

(c)

Fig. 6. Relevant vertices in the tree: $y^{\prime}$ is the projection of $y$ onto $P(w, r)$. 